\title{
Luxationen und Luxationsfrakturen des Kniegelenks
}

Tobias Claus Drenck, Maximilian Heitmann, Ralph Akoto, Achim Preiss, Jannik Frings, Matthias Krause, Karl-Heinz Frosch

\section{Definition, Häufigkeit und Verletzungsmechanismus}

Kniegelenkluxationen und -luxationsfrakturen zählen zu den schwersten Gelenkverletzungen überhaupt. Die Verletzung definiert sich zunächst über einen Verlust der tibiofemoralen Gelenkintegrität bzw. einer zusätzlich knöchernen Beteiligung. Trotz deutlicher Fortschritte in der Behandlung und dem Aufkommen neuer Therapiemöglichkeiten stellen diese Verletzungen für Orthopäden und Unfallchirurgen eine Herausforderung dar. Dies begründet sich auch in der Tatsache, dass es sich bei der Kniegelenkluxation um eine relativ seltene Verletzung handelt; die Häufigkeit wird mit $0,02-0,2 \%$ aller muskuloskeletalen Verletzungen und etwa 0,2-0,3\% aller Gelenkluxationen angegeben [1,2]. Etwa 50\% aller Kniegelenkluxationen reponieren sich spontan [3]. Die Vielfalt der Verletzung erfordert die interdisziplinäre Zusammenarbeit verschiedener Fachrichtungen zur knöchernen, ligamentären sowie Gefäß- und Nervenrekonstruktion. Der Verletzungsmechanismus der Kniegelenkluxation unterscheidet sich in 2 Subtypen: Etwa die Hälfte ereignet sich im Rahmen von Hochrasanztraumata wie z.B. bei Verkehrsunfällen. 14-44\% der Verletzungen resultieren aus einem Polytrauma, Männer sind etwa 4-mal häufiger betroffen. Demgegenüber finden sich sog. Low-Velocityund Ultra-low-Velocity-Verletzungsmechanismen (ca. $10 \%)$, in diesem Zusammenhang spielt die Adipositas eine große Rolle. Ridley et al. zeigten 2014, dass die Komplikationsrate mit jedem BMI-Punkt um 9,2\% zunimmt $[1,3,4]$. In einer großen Untersuchung von Green und Allen war die Luxation der Tibia nach anterior mit $40 \%$ die am häufigsten beobachtete Form unter 245 Luxationen [3]. Eine Kadaverstudie von Kenney zeigte dabei ein Hyperextensionstrauma als typischen Verletzungsmechanismus, mit zunehmender Hyperextension kommt es zu einem Versagen der dorsalen Kapsel sowie zur Ruptur des vorderen und hinteren Kreuzbandes und schlussendlich bei $50^{\circ}$ zu einem obligaten Zerreißen der A. poplitea [3]. Die hintere Luxation der Tibia ist mit 33\% der zweithäufigste Typ und Folge einer direkten nach dorsal gerichteten Krafteinwirkung auf den Unterschenkel. Typischerweise findet sich diese Verletzung bei Verkehrsunfällen mit Anprall des Armaturenbrettes auf das gebeugte Kniegelenk „Dashboard Injury“ [3].

\section{Klassifikation}

In der klinischen Praxis hat sich die Klassifikation nach Schenck durchgesetzt, die das Verletzungsmuster und somit die resultierende Instabilität beschreibt. Wascher modifizierte die Klassifikation um die Luxationsfrakturen (Typ V, siehe $>$ Tab. 1). In der Klassifikation nach Schenk handelt es sich bei der Verletzung Typ III mit Ruptur des vorderen und hinteren Kreuzbandes sowie des medialen oder lateralen Seitenbandes um die häufigste Form der Luxation. Die begleitende Verletzung von Nerv und/oder Gefäß wird gesondert klassifiziert und hat elementaren Einfluss auf das Therapieregime wie auch auf das klinische Outcome.

Merke

Kniegelenkluxationen können auch bei geringer Krafteinwirkung auftreten, häufig liegt eine spontane Reposition vor. Die Verletzung darf jedoch aufgrund potenzieller Gefäß-/Nervenverletzungen keinesfalls übersehen oder unterschätzt werden.

\section{Diagnostik}

Die initiale klinische Diagnostik und Untersuchung erfolgt, wie nach jedem Trauma üblich, nach dem ATLS ${ }^{\circledR}$ Algorithmus (Advanced Trauma Life Support). Die differenzierte Untersuchung des Kniegelenks erfolgt erst nach der Behandlung von lebensbedrohlichen Verletzun-

- Tab. 1 Kniegelenkluxation: Einteilung nach Schenk und modifiziert nach Wascher.

Typ I $\quad$ Ruptur eines Kreuzbandes (VKB/HKB) und mindestens eines Kollateralbandes (MCL/LCL)

\begin{tabular}{|l|l|}
\hline Typ II & Ruptur beider Kreuzbänder (VKB/HKB) \\
\hline Typ III lateral & Ruptur beider Kreuzbänder (VKB/HKB) und des LCL \\
\hline Typ III medial & Ruptur beider Kreuzbänder (VKB/HKB) und des MCL \\
\hline Typ IV & $\begin{array}{l}\text { Ruptur beider Kreuzbänder (VKB/HKB) und beider } \\
\text { Kollateralbänder (MCL/LCL) }\end{array}$ \\
\hline Typ V & Luxationsfraktur \\
\hline N & Nervenläsion \\
\hline V & Gefäßläsion \\
\hline
\end{tabular}

VKB = vorderes Kreuzband; $\mathrm{HKB}$ = hinteres Kreuzband; $\mathrm{MCL}$ = mediales Kollateralband (Innenband); LCL = laterales Kollateralband (Außenband) 


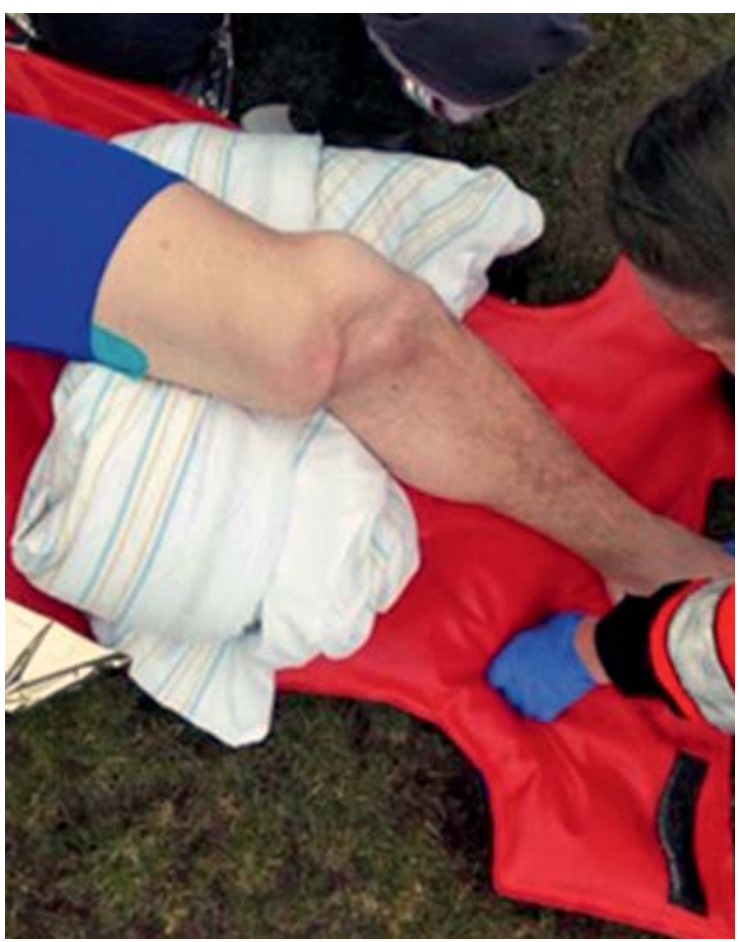

- Abb. 1 Ein luxiertes Kniegelenk am Unfallort: Sofern möglich sollte eine präklinische Reposition erfolgen.

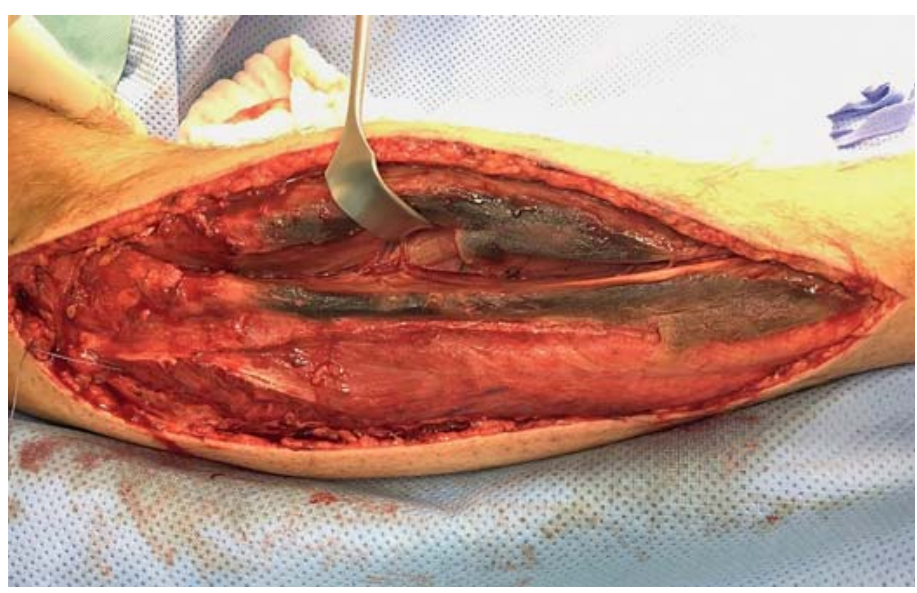

- Abb. 2 Zu den schwersten Begleitverletzungen einer Knieluxation gehört das Kompartmentsyndrom. Wird dieses nicht frühzeitig erkannt, droht ein erheblicher Funktionsverlust der Extremität bis hin zur Amputation.

gen im Secondary Survey. Da bei Gefäß- und Nervenverletzungen im Falle einer Knieluxation ein Verlust der Extremität droht, ist eine differenzierte Untersuchung des Kniegelenks zwingend erforderlich. Inspiziert werden die vorliegenden Weichteilverletzungen bei offener oder geschlossener Luxation und/oder Fraktur, die Achsfehlstellungen und Gelenkkongruenz. Befindet sich das Gelenk in grober Fehlstellung, sollte es umgehend ggf. präklinisch unter Analgosedierung reponiert werden
( $\triangleright$ Abb. 1). Das Vorliegen eines Kniegelenkergusses macht einen Kniebinnenschaden hochwahrscheinlich, ein fehlender Erguss schließt diesen jedoch nicht sicher aus. Auch oberflächige Kontusionen oder Schürfungen können z. B. für die komplexe Planung der operativen Zugangswege einer späteren Versorgung von entscheidender Wichtigkeit sein. Je nach Ausmaß der Verletzung kann sich schon früh ein Kompartmentsyndrom manifestieren ( $\checkmark$ Abb. 2). Bei beatmeten Patienten mit eingeschränkter klinischer Beurteilbarkeit kann eine Kompartmentdruckmessung Aufschluss geben, entscheidend ist jedoch der klinische Aspekt. In der Akutsituation dürfen Gefäß-Nerven-Verletzungen nicht übersehen werden. Die Häufigkeit einer Gefäßverletzung bei Knieluxation wird in der Literatur mit etwa $20 \%$ angegeben, bei dorsalen Luxationen ist eine Gefäßverletzung deutlich wahrscheinlicher [1]. In der Fallserie von Green und Allen mit 245 Knieluxationen präsentierten sich 44\% der dorsalen Luxationen sowie 39\% der anterioren Luxationen mit Gefäßverletzungen [3,5]. Je nach Studie werden Amputationsraten bis zu $12 \%$ beschrieben [1]. Der Fokus der klinischen Untersuchung sollte in der Akutphase in der Evaluation der neurovaskulären Strukturen liegen. Eine differenzierte ligamentäre Untersuchung ist in der Akutphase häufig nicht möglich; eine hochgradige multidirektionale Instabilität lässt sich jedoch meist leicht feststellen. Die klinische Untersuchung umfasst daher zwingend die Palpation der peripheren Gefäße. Intakte Pulse im Bereich $\operatorname{der}$ A. dorsalis pedis lateral der Sehne des M. extensor hallucis longus sowie der $A$. tibialis posterior hinter dem Innenknöchel schließen jedoch okkulte Gefäßverletzungen wie eine Intimaläsion nicht aus. Die Diagnostik wird häufig durch eine Messung des ABI (Ankle Brachial Pressure Index) als einfache technische Untersuchung ergänzt. In einer Studie von Mills et al. zeigte sich eine hohe Spezifität und Sensitivität von über 95\% für die Feststellung einer behandlungswürdigen Gefäßpathologie [1,6].

Merke

Zu den schwersten Begleitverletzungen einer Knieluxation gehört das Kompartmentsyndrom. Wird dieses nicht frühzeitig erkannt, droht ein erheblicher Funktionsverlust der Extremität bis hin zur Amputation.

\section{Bildgebende Diagnostik}

Native Röntgendiagnostik

Bereits im Schockraum oder nach Durchführung der Primärdiagnostik sollte ein Standardröntgen in 2 Ebenen durchgeführt werden, so kann ggf. ein Repositionsergebnis und knöcherne Begleitverletzungen gesichert werden ( Abb. 3).

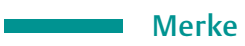

Bei jeder Kniegelenkluxation sollte eine bildgebende Darstellung der Gefäße erfolgen. 


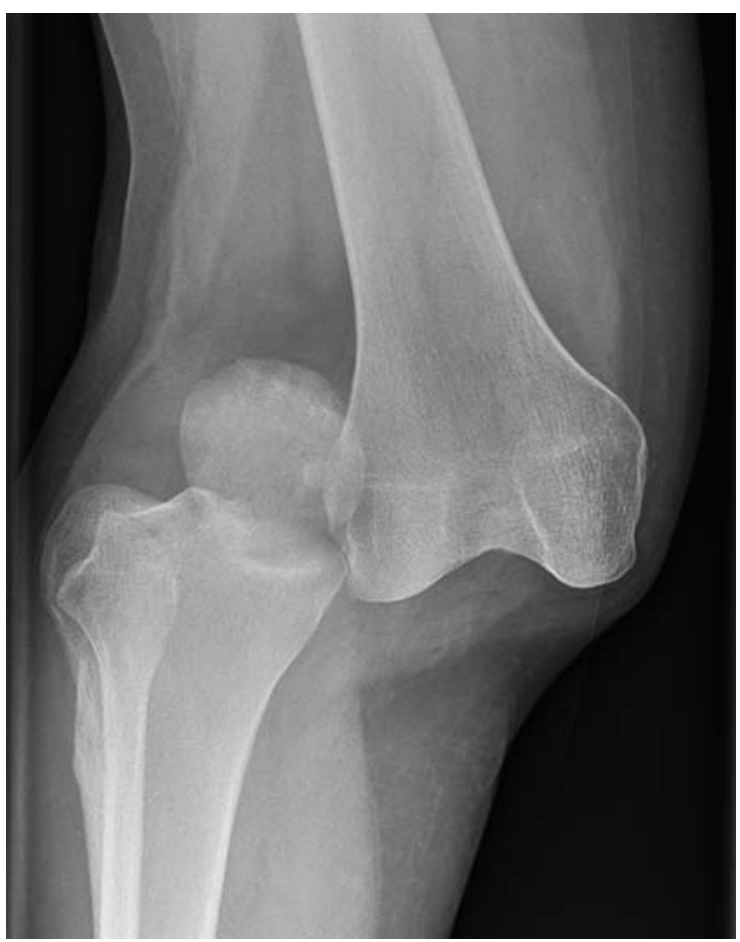

Abb. 3 Ein a.-p. Röntgenbild eines nach medial luxierten Kniegelenks.

\section{Doppler-Sonografie}

Die Doppler-Sonografie ist eine nicht invasive Möglichkeit, Gefäßverletzungen darzustellen, wenn durch eine Niereninsuffizienz eine CT-Angiografie nicht möglich ist. Limitierend sind fehlende Erfahrungen des Untersuchers, um auch kleine Intimaläsionen zu detektieren. Ferner ist der Zeitfaktor ein wesentlicher Aspekt, eine vollständige und gründliche Diagnostik dauert hier deutlicher länger als eine Kontrastmitteldarstellung. Insbesondere im Falle eines Polytraumas sind derart zeitaufwendige Untersuchungen nicht immer mit dem weiteren Management zu vereinen.

\section{CT- und MRT-Angiografie}

Der Goldstandard der Gefäßdiagnostik ist eine Darstellung mithilfe eines Kontrastmittels. Die Dringlichkeit verdeutlicht eine Studie von Barnes et al., in der trotz initial unauffälligem Pulsstatus in 9\% der Fälle eine Intimaverletzung der A. poplitea festgestellt wurde $[1,7]$. In unserer Klinik führen wir bei jeder Kniegelenkluxation deshalb eine bildgebende Diagnostik der Gefäße durch ( Abb. 4). Aufgrund der schnellen Durchführbarkeit und Verfügbarkeit hat die CT-Diagnostik Vorteile gegenüber der Bildgebung mittels MRT. Bei knöcherner Beteiligung und Frakturen führen wird grundsätzlich eine $C T$ des Kniegelenks durch.

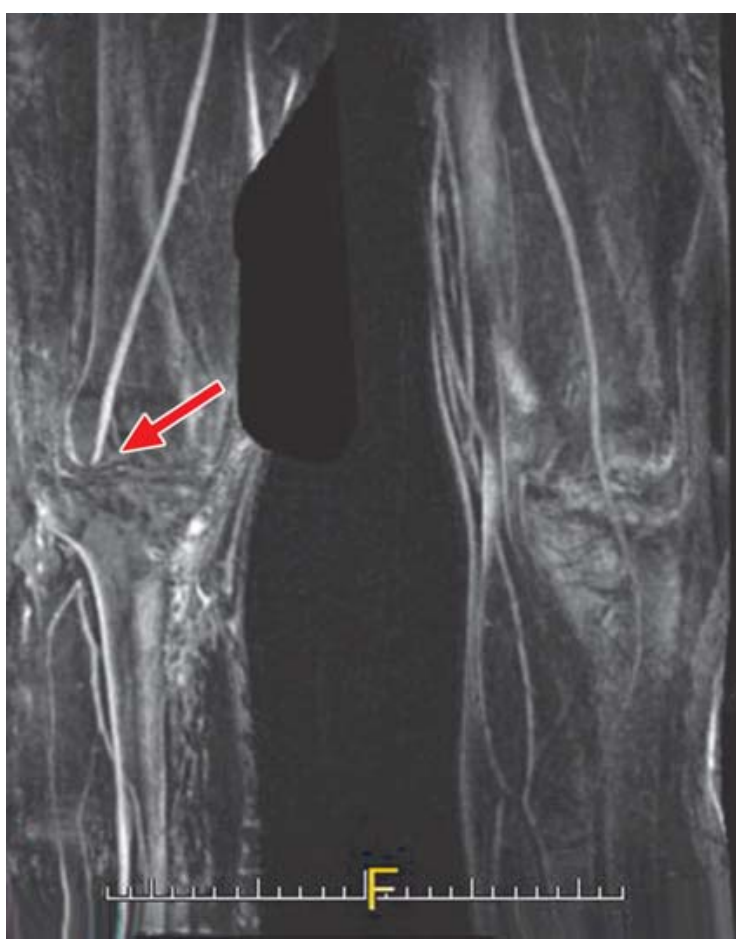

- Abb. 4 Zeigt eine Gefäßverletzung in einer MR-Angiografie mit Abbruch der Kontrastierung in der A. poplitea auf Höhe des Gelenkspaltes.

\section{Bildgebung der operativen Planung}

Nachfolgend an die akute Bildgebung stellt das MRT den Goldstandard zur Beurteilung ligamentärer Verletzungen dar, je nach Studie können diese mit einer Genauigkeit von $85-100 \%$ festgestellt werden [1]. Ferner können Begleitverletzungen des Meniskus oder traumatische Knorpelschäden detektiert werden. Wir empfehlen die Durchführung einer MRT nach jeder Kniegelenkluxation, problematisch ist die Bildgebung im Falle eines anliegenden Fixateur externe, bevorzugt sollten daher MR-fähige Fixateursysteme Verwendung finden ( $\triangleright$ Abb. 5).

Merke

Jedes luxierte Gelenk sollte zur Beurteilung von Begleitverletzungen mittels MRT dargestellt werden.

Im Falle einer chronischen Instabilität nimmt die Aussagekraft der MRT-Bildgebung ab. Die klinische Untersuchung und genaue Evaluation der Instabilität tritt in den Vordergrund, ergänzt wird die Untersuchung durch gehaltene Röntgenaufnahmen im TELOS ${ }^{-G e r a ̈ t . ~ I n s b e s o n-~}$ dere für Verletzungen des HKB oder kombinierte posterolaterale Verletzungen bieten gehaltene Aufnahmen die Möglichkeit, die a.-p. Translation im Seitenvergleich zu messen und das Maß der Instabilität zu beurteilen. Die Komplexität der Verletzung erfordert in einigen Fällen ergänzende Diagnostik durch eine Ganzbeinstandaufnah- 


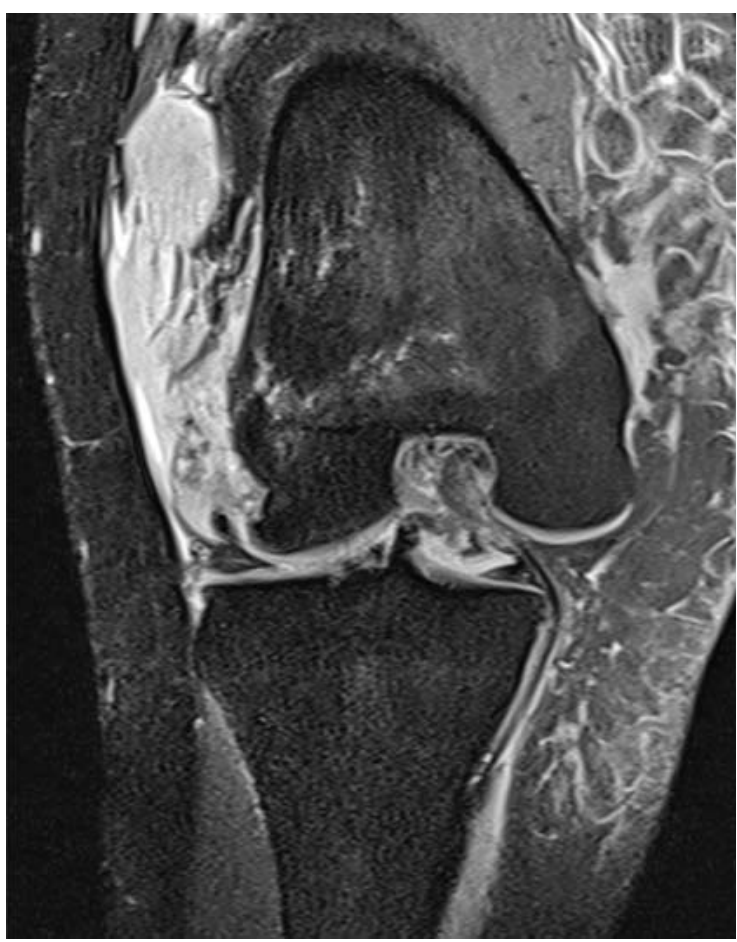

- Abb. 5 Jedes luxierte Gelenk sollte zur Beurteilung von Begleitverletzungen mittels MRT dargestellt werden.

me sowie eine seitliche Aufnahme des Unterschenkels zur Bestimmung des tibialen Slope.

\section{Therapie}

\section{Merke}

Die konservative Therapie einer Kniegelenkluxation zeigt in etwa $80 \%$ unbefriedigende Ergebnisse und ist der operativen Therapie unterlegen [1].

\section{Operativ oder konservativ, ein- oder zweizeitiges Vorgehen?}

Das Aufkommen moderner Implantate mit hoch reißfesten Fäden, Knochenankern und Plattenosteosynthesen ermöglicht heutzutage innovative operative Versorgungstechniken. Die operative Therapie akuter wie auch chronischer Verletzungen ist in der aktuellen Studienlage der konservativen Therapie weit überlegen. So zeigten Untersuchungen von Levy et al. und Frosch et al. schlechte Ergebnisse nach konservativer Therapie in 70-80\% der Fälle. Die operative Therapie konnte hingegen in etwa $80 \%$ der Fälle ein gutes oder sehr gutes subjektives und objektives Ergebnis erzielen [1,8,9].

Zur operativen Therapie werden in der Literatur 2 unterschiedliche Vorgehensweisen kontrovers diskutiert. Weit verbreitet ist ein zweizeitiges Vorgehen: In der Akutphase werden zunächst die Kollateralbänder genäht oder mittels Bandplastik rekonstruiert. Anschließend erfolgt in einer weiteren Operation die Versorgung des zentralen Pfeilers mit Bandplastiken des VKB und HKB. Das einzeitige Vorgehen durch Naht aller rupturierten Bänder zeigt in der Literatur gleich gute Ergebnisse [1]. Heitmann et al. zeigten 2014, dass durch eine Fadenaugmentation die Primärstabilität einer Bandnaht signifikant erhöht werden kann [10]. Entscheidend für eine erfolgreiche primäre Bandnaht ist der Zeitpunkt der Operation; Richter et al. konnten die besten Ergebnisse bei einer frühen operativen Versorgung in der 1. Woche feststellen [11]. Ein früher Versorgungszeitpunkt birgt jedoch die Gefahr einer postoperativen Arthrofibrose mit nachfolgender Bewegungseinschränkung [1].

\section{Operatives Vorgehen Kollateralbänder}

Mediales Kollateralband

Das mediale Kollateralband ist der primäre Stabilisator gegen valgisierende Kräfte am Kniegelenk. Biomechanisch und anatomisch besteht es aus 3 Anteilen: dem oberflächigen Teil (sMCL), dem tiefen Anteil (dMCL) sowie dem hinteren Schrägband (POL). Im Falle einer Schenk-III-Luxation kommt es zu einer Zerreißung des Innenbandes, meist im Sinne einer Grad-III-Verletzung nach Hughston. Komplette Rupturen des Innenbandes sollten dabei primär genäht werden, etwa $70 \%$ aller Innenbandverletzungen befinden sich am knöchernen Ansatz, hier ist eine Refixierung mittels Fadenanker zu empfehlen [1]. Die Primärstabilität kann dabei durch eine zusätzliche Fadenaugmentation signifikant erhöht werden [10]. Ist der Bandapparat ausgefasert oder retrahiert, ist eine primäre Naht häufig nicht erfolgversprechend, hier sollte ggf. bereits primär eine Bandplastik erfolgen. In der Literatur finden sich verschiedene Techniken zur Rekonstruktion des Innenbandes [12]. Nach unserer Erfahrung lassen sich die besten Ergebnisse hinsichtlich Stabilität und Funktionalität mit einer anatomischen Rekonstruktion beider Bündel (sMCL und POL) erzielen. Preiss et al. beschrieben hierzu eine minimalinvasive 2-BündelTechnik, um sowohl das SMCL als auch das POL zu ersetzen. Sofern möglich, wird dabei die kontralaterale M.-semitendinosus-Sehne verwendet, um eine weitere Schwächung der dynamischen medialen Stabilisatoren am verletzen Kniegelenk zu vermeiden [12].

\section{Laterales Kollateralband}

Verletzungen des lateralen Bandapparates sind mit etwa 7-17\% insgesamt seltener als mediale Bandverletzungen. Der außenseitige Bandapparat sichert das Kniegelenk mit seinen passiven und aktiven Stabilisatoren gegen Varusstress und Außenrotation. Eine Verletzung des LCL resultiert primär in einer varischen Instabilität. Ist zusätzlich das Lig. popliteofibulare (Lig. PFL) betroffen oder die Popliteussehne abgerissen, zeigt sich eine kombinierte Rotationsinstabilität [13]. Das komplexe Zusammenspiel des LCL, HKB und Lig. PFL machen eine alleinige Bandnaht ungleich schwieriger als am medialen Bandkomplex. Zeigt sich die Sehne des M. popliteus am femoralen An- 
satz verletzt, sollte eine Refixation mittels Fadenanker erfolgen. Unsere Erfahrungen aus der Versorgung von über 200 Knieluxationen zeigen, dass sich am lateralen Kniegelenk durch eine alleinige primäre Bandnaht häufig keine zufriedenstellende Stabilität erreichen lässt. Im Gegensatz zum medialen Bandapparat wird in der Literatur häufig von schlechten Ergebnissen nach alleiniger Naht des Außenbandkomplexes berichtet. Stannard et al. zeigten 2005 eine deutlich höhere Versagensquote bei Patienten, bei denen eine alleinige Bandnaht erfolgte ( 9 vs. $37 \%)$ [14]. Im eigenen Vorgehen kombinieren wir daher die primäre Bandnaht mit einer Bandplastik. Im chronischen Fall der Verletzung ist eine Versorgung mittels Bandplastik obligat. In der Literatur findet sich eine Vielzahl an Techniken zur Rekonstruktion der lateralen und posterolateralen Bänder. Am häufigsten wird bisher die Bandplastik nach Larson eingesetzt, hierzu wird eine Hamstringsehne durch die proximale Fibula geführt und zwischen den femoralen Insertionspunkten des LCL und der Popliteussehne befestigt. Die Rekonstruktion ist der Versuch, durch einen extraanatomischen Verlauf die biomechanischen Eigenschaften des LCL und PFL zu kompensieren. Biomechanische Studien haben jedoch gezeigt, dass sich die Rotationsstabilität auf diese Weise nur schlecht adressieren lässt. Komplexere Rekonstruktionen nach LaPrade [15] mit einem Popliteus-Bypass oder nach Arciero [16] mit 2 femoralen Bohrkanälen erzielen bessere Ergebnisse [1]. Bei jedem operativen Zugang zur posterolateralen Ecke und unabhängig von der gewählten Rekonstruktion empfehlen wir eine Darstellung und Dekompression des N. peroneus am Hinterrand des M. biceps femoris. Eine Ausnahme bilden rein arthroskopische Techniken, die dies nicht erfordern ( $>$ Abb. 6).

\section{Operatives Vorgehen zentraler Pfeiler}

Die Therapie des zentralen Pfeilers wird in der Literatur kontrovers diskutiert. Die verschiedenen Auffassungen resultieren meist aus der Angst vor einer Arthrofibrose. Frühe Studien zeigten diesbezüglich hohe Raten von bis zu 29\%. Die resultierende Bewegungseinschränkung und die damit verbundenen chronischen Schmerzen stellen für den Patienten einen hohen Verlust an Lebensqualität dar [1]. Aufgrund dieser Bedenken sprechen sich noch heute Autoren für eine verzögerte bzw. zweizeitige operative Therapie aus. Die aktuelle Literatur zeigt jedoch eine deutliche Überlegenheit der frühzeitigen Operation [1]. Im Falle eines zweizeitigen Vorgehens wird nach ausgeheilter primärer Bandnaht der Kollateralbänder eine Bandplastik des VKB und HKB vorgenommen. Dies unterscheidet sich im grundsätzlichen Vorgehen nicht von den üblichen Techniken der Kreuzbandplastiken mit verschiedenen Sehnen und Fixationstechniken. Im Falle eines zweizeitigen Vorgehens sollte entschieden darauf geachtet werden, dass die Kollateralbänder ausreichend stabil ausgeheilt sind. Eine verbliebende mediale oder laterale Instabilität wird mit hoher Wahrscheinlichkeit zu einem Transplantatversagen führen.

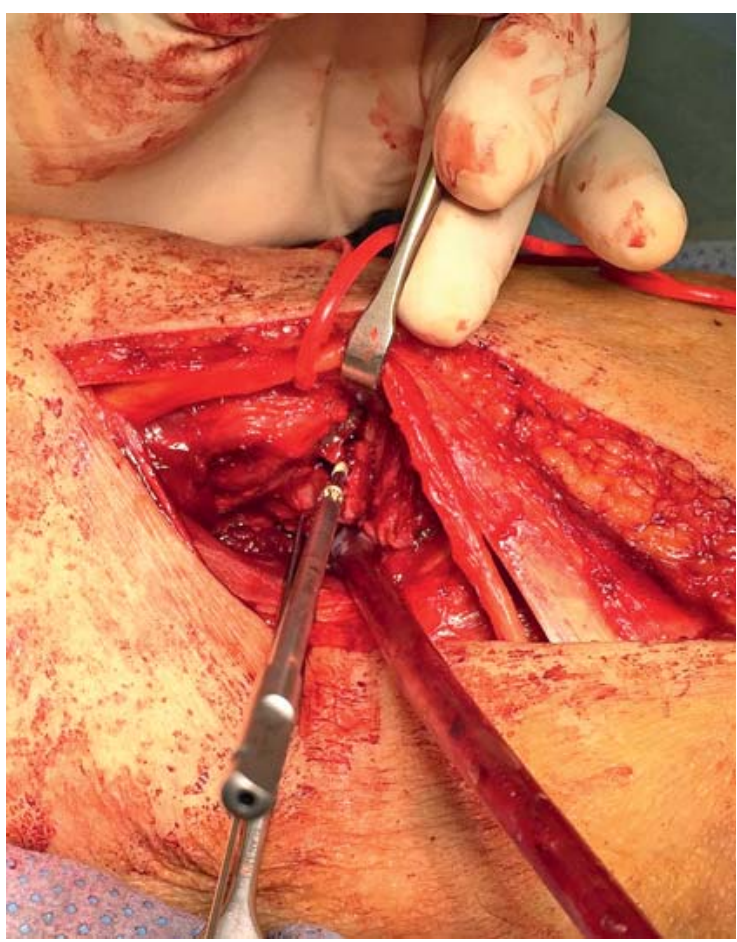

- Abb. 6 Bei jedem posterolateralen Zugang zum Kniegelenk empfehlen wir die Darstellung und Dekompression des N. peroneus (angeschlungen).

\section{Ligament Bracing zur Therapie des zentralen Pfeilers}

Das Ligament Bracing wurde als Versorgungsstrategie der frischen Kniegelenkluxation 2014 von Heitmann und Frosch entwickelt [10]. Hierzu wurde die Technik einer primären Bandnaht durch die zusätzliche Augmentation mittels hoch reißfester Fäden weiterentwickelt ( $\boldsymbol{A}$ Abb. 7 ).

Nach der obligaten Narkoseuntersuchung des Kniegelenks erfolgt eine Arthroskopie, um ggf. Begleitverletzungen zu detektieren und ggf. auch primär zu behandeln (z.B. Meniskusverletzungen). Anschließend erfolgt eine mediale Arthrotomie, die Patella wird dabei nach lateral gehalten, sodass die Notch mit den Kreuzbändern eingesehen werden kann ( $\bullet$ Abb. 7). Die Kreuzbandenden sollten für eine Naht geeignet sein, ein sehr ausgefasertes Ende macht eine suffiziente Naht unmöglich. Die Enden von VKB und HKB werden anschließend mit festen Fäden durchflochten und somit armiert. Mit arthroskopischen Zielgeräten werden nun 4,5-mm-Tunnel in die femoralen und tibialen Insertionen der Kreuzbänder gebohrt. Die Fäden werden anschließend zusammen mit einem hoch reißfesten Faden zur Augmentation durch die Bohrkanäle ausgeleitet und über Flip-Buttons extrakortikal verknotet. Die Gelenkkongruenz ist in der Sagittalebene häufig schwer einzustellen, daher sollte die Fixierung intraoperativ unter seitlicher fluoroskopischer Kontrolle der Gegenseite erfolgen. Das Ergebnis ist eine augmentierte Kreuzbandnaht, die eine signifikant höhere Primärstabili- 


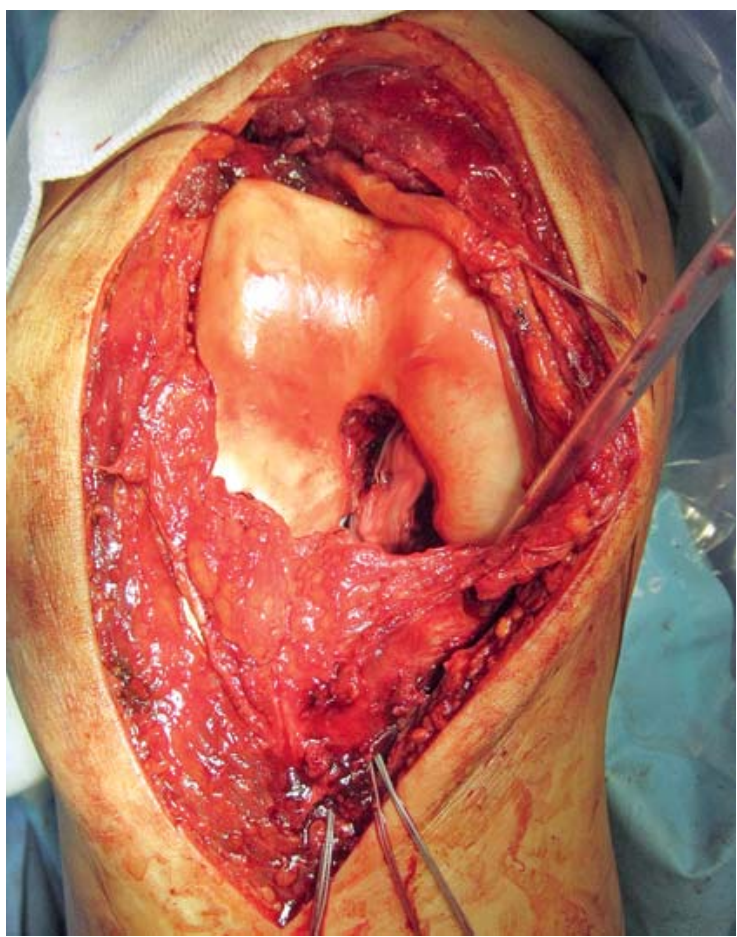

Abb. 7 Ligament Bracing: Durch eine mediale Arthrotomie werden die Kreuzbänder genäht und durch einen hochreißfesten Faden verstärkt.

tät bietet als die alleinige Naht. In gleicher Sitzung werden auch die Kollateralbänder durch Bandnähte bzw. Bandrekonstruktionen versorgt.

\section{Luxationsfrakturen}

Die Kniegelenkluxation Typ V nach Schenk definiert sich als kombinierte knöcherne und ligamentäre Verletzung und erhöht die Komplexität der Versorgung deutlich $(\triangleright$ Abb. 8). Eine weitere gängige Klassifikation erfolgt nach Moore und unterteilt die Kniegelenkluxationsfrakturen in 5 Typen [17]. Die Komplexität der Fraktur beeinflusst das Behandlungsergebnis maßgeblich, je nach Literatur werden Arthroseraten von bis zu $44 \%$ beschrieben [18]. Die Krafteinwirkung resultiert meist in dem Versagen der Bänder oder der knöchernen Strukturen, es kann auch zu kombinierten Verletzungen kommen. In einer großen Studie von 315 Patienten mit Tibiakopffrakturen zeigten 21,9\% eine begleitende Bandverletzung. Dabei wurden bei 49,3\% isolierte Schädigungen des medialen, 31,9\% des lateralen Seitenbandes sowie bei 5,8\% eine Verletzung des vorderen Kreuzbandes gefunden. Eine begleitende Bandverletzung findet sich demnach häufig, eine vollständige Gelenkluxation mit kombinierter Tibiakopffraktur stellt jedoch eine seltene Verletzung dar [17]. Das initiale Vorgehen unterscheidet sich nicht von der reinen Luxation, auch hier liegt der Fokus auf Gefäß-/Nervenverletzungen und den Weichteilverhältnissen. Bei Frakturen, die beide Kondylen des Tibiaplateaus

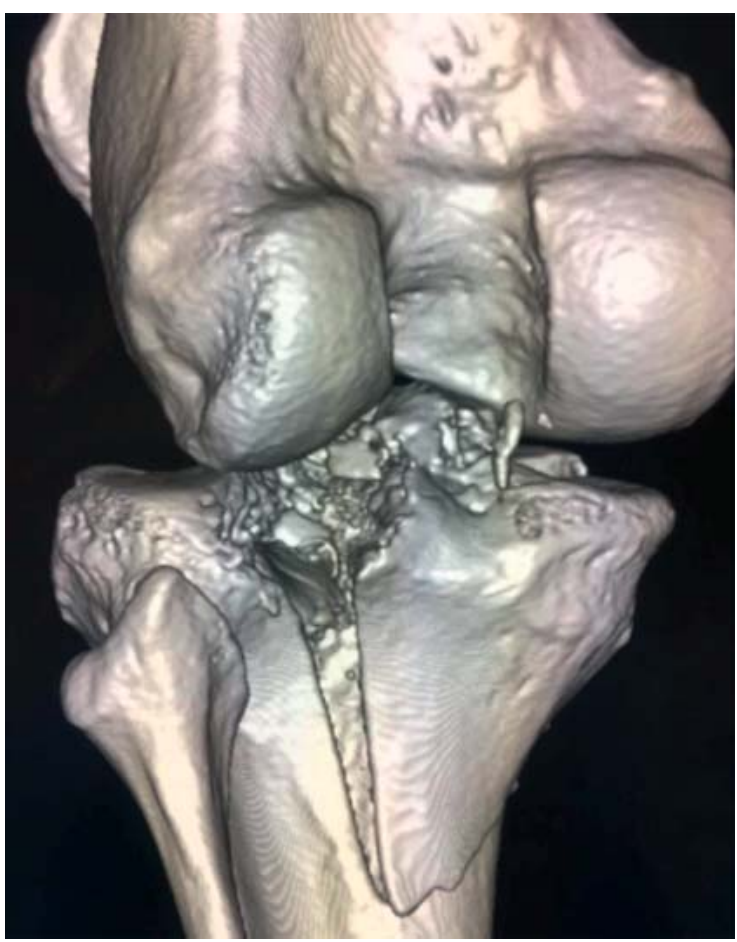

- Abb. 8 CT-Darstellung einer Moore-Typ-II-Luxationsfraktur.

betreffen (Typ C nach AO), kann die Länge der Extremität durch die Frakturmorphologie nicht gestützt werden. In diesem Fall sollte die Fraktur initial im gelenkübergreifenden Fixateur externe stabilisiert werden. Bei einfacheren Luxationsfrakturen ist je nach Zustand der Weichteile (Kompartment, Gefäß-/Nervenverletzung) die Ruhigstellung in einer Orthese meist möglich. Bei kombinierten ligamentären und knöchernen Verletzungen empfehlen die meisten Autoren die Fokussierung auf die bestmögliche knöcherne Wiederherstellung mittels Platten- und Schraubenosteosynthese. In Abhängigkeit von der Frakturmorphologie ist eine zeitgleiche Bandnaht jedoch grundsätzlich möglich. Krause et al. beschrieben 2017 eine 10-Segment-Klassifikation zur Einteilung und Planung einer Osteosynthese bei komplexen Tibiakopffrakturen [18]. Bei den Avulsionsfrakturen (A1 nach AO) handelt es sich hingegen nur selten um vollständige Luxationen; knöcherne Ausrisse der Eminentia, der tibialen HKBInsertion oder der proximalen Fibula können meist durch eine primäre Verschraubung oder transossäre Ausziehnähte direkt stabilisiert werden. Knöcherne HKB-Ausrisse können durch einen minimalinvasiven Zugang zum dorsalen Tibiakopf reponiert und mittels kanülierter Schrauben versorgt werden [19]. Eine häufige Frakturform ist die vom Typ Moore II mit Beteiligung der gesamten Kondyle, in diesen Fällen kann die gesamte Versorgung meist in Bauchlage über einen dorsomedialen Zugang erfolgen ( Abb. 9). 


\section{Nachbehandlung}

\section{Merke}

Die Nachbehandlung sollte individuell erfolgen und ist eine Gratwanderung zwischen resultierender Instabilität oder bleibender Bewegungseinschränkung. Beides ist regelhaft durch eine optimale Nachbehandlung vermeidbar.

Aufgrund der Vielfältigkeit der Verletzung muss jede Nachbehandlungsstrategie individuell gewählt werden; das schwächste Glied der Rekonstruktion bestimmt dabei das Ausmaß. Die Nachbehandlung muss dabei dem Verletzungsmuster, der spezifischen Versorgung und der Konstitution des Patienten angepasst werden. Jede Nachbehandlung einer Kniegelenkluxation steht in einem Gleichgewicht zwischen persistierender Instabilität und resultierender Bewegungseinschränkung. In der Regel erfolgt postoperativ eine Behandlung durch eine Hartrahmenorthese von 6-12 Wochen mit individueller Limitierung des Bewegungsausmaßes. Ist die Extremität durch eine hochgradige Adipositas für eine Orthesenbehandlung ungeeignet, empfehlen die Autoren die Ruhigstellung im Fixateur externe für 4-6 Wochen, um die Rekonstruktion zu schützen. Der Belastungsaufbau variiert ebenfalls zwischen den unterschiedlichen Verletzungsmustern. Im Falle einer primären Bandnaht wählen wir eine Teilbelastung mit $20 \mathrm{~kg}$ Körpergewicht für 6 Wochen, hingegen benötigen komplexe Tibiakopfimpressionsfrakturen eine Teilbelastung unter Sohlenkontakt von bis zu 12 Wochen. Aufgrund des hohen Risikos einer Arthrofibrose sollte man sofern möglich eine offensive Nachbehandlung wählen: So kann nach einer primären Bandnaht bereits nach wenigen Tagen in der geschlossenen Kette z.B. mit Kniebeugen trainiert werden. Die Nachbehandlung sollte initial engmaschig kontrolliert und ggf. angepasst werden, sodass sich die Patienten alle 14 Tage ärztlich vorstellen sollten.

\section{Fazit}

- Die Kniegelenkluxation ist eine seltene, aber schwere Verletzung und ist meist Folge von Hochrasanzverletzungen. Sie kann jedoch auch als Ultra-low-VelocityVerletzung bei Adipositas auftreten.

- Die Erstmaßnahmen beinhalten eine ggf. präklinische Reposition des Gelenks unter Analgosedierung.

- Die initiale Diagnostik erfordert ein Standardröntgen, entscheidend sind jedoch begleitende Gefäß- und Nervenverletzungen: Eine Gefäßdarstellung sollte daher obligat erfolgen.

- Die konservative Therapie von Kniegelenkluxationen führt zu schlechten Ergebnissen, die operative Therapie ist daher zu bevorzugen.

- Eine primäre Bandnaht aller Bänder, ggf. mit Augmentation, sollte innerhalb der ersten 10 Tage erfolgen. Alternativ kann ein zweizeitiges Vorgehen gewählt

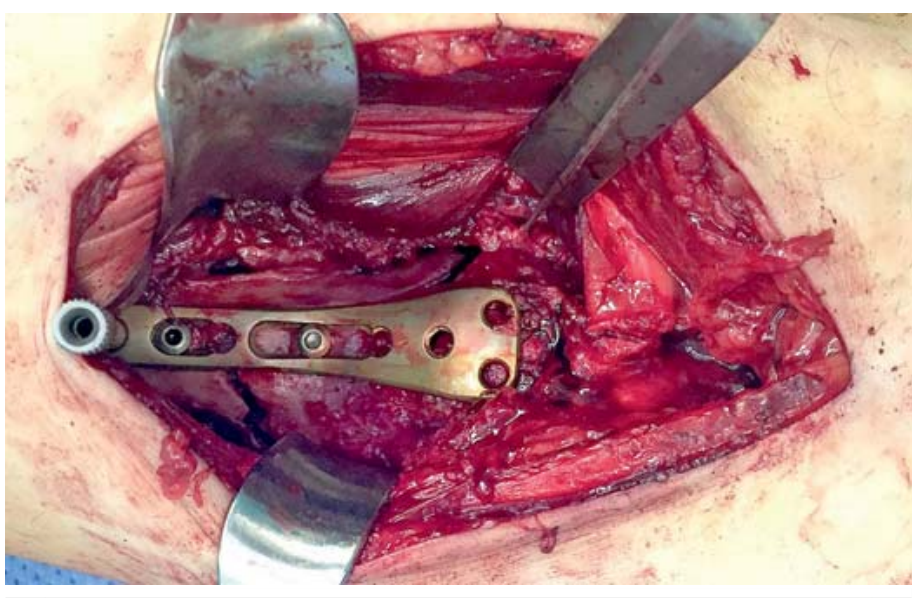

Abb. 9 Operative Versorgung einer Moore-Typ-II-Luxationsfraktur über einen dorsomedialen Zugang in Bauchlage.

werden, bei dem die Versorgung der Seitenbänder vor dem Ersatz der Kreuzbänder erfolgt.

- Zur Rekonstruktion der Bänder sollten anatomische Techniken bevorzugt werden.

- In der Nachbehandlung muss auf eine beginnende Arthrofibrose und eine chronische Instabilität geachtet werden, die Patienten sollten daher engmaschig nachkontrolliert werden.

- Durch geeignete OP-Techniken lassen sich bei den meisten Patienten gute bis sehr gute klinische Ergebnisse erzielen.

\section{Autorinnen/Autoren}

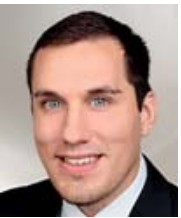

\section{Tobias Claus Drenck}

Dr. med., Assistenzarzt, Asklepios Klinik St. Georg, Chirurgisch-Traumatologisches Zentrum, Hamburg

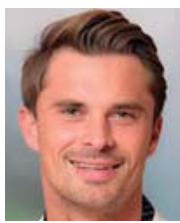

\section{Maximilian Heitmann}

Dr. med., Leitender Oberarzt Sportorthopädie, Argon Orthopädie Hamburg, Helios Endoklinik

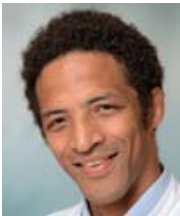

\section{Ralph Akoto}

Dr. med., Leitender Oberarzt, Sektion Knieund Schulterchirurgie, Sporttraumatologie, Asklepios Klinik St. Georg, Chirurgisch-Traumatologisches Zentrum, Hamburg

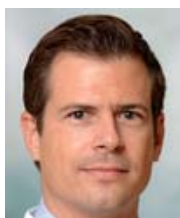

\section{Achim Preiss}

Dr. med., Oberarzt, Chirurgisch-Traumatologisches Zentrum, Knie- und Sprunggelenkschirurgie, Sporttraumatologie, Asklepios Klinik St. Georg, Hamburg 


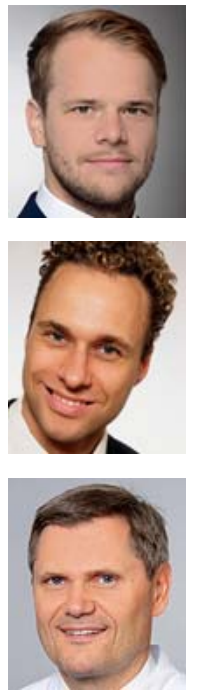

\section{Jannik Frings}

Dr. med., Assistenzarzt, Klinik für Unfall-, Handund Wiederherstellungschirurgie, Universitätsklinikum Hamburg-Eppendorf

\section{Matthias Krause}

PD Dr. med., Assistenzarzt, Klinik für Unfall-, Hand- und Wiederherstellungschirurgie, Universitätsklinikum Hamburg-Eppendorf

\section{Karl-Heinz Frosch}

Univ.-Prof. Dr. med., Direktor, Klinik für Unfall-, Hand- und Wiederherstellungschirurgie, Universitätsklinikum Hamburg-Eppendorf

\section{Korrespondenzadresse}

Prof. Dr. med. Karl-Heinz Frosch

Direktor

Klinik und Poliklinik für Unfall-, Hand- und Wieder-

herstellungschirurgie

Universitätsklinikum Hamburg-Eppendorf

Martinistraße 52

20246 Hamburg

Tel.: 040/7410-53459

Fax: 040/7410-54569

k.frosch@uke.de

\section{Interessenkonflikt}

Die Autoren haben keinen Interessenkonflikt.

\section{Literatur}

[1] Rixen D, Schoepp C, Tingart M. Kniechirurgie. München: Urban \& Fischer; 2016

[2] Shafizadeh ST, Bouillon B, Naendrup JH et al. Behandlung der akuten Kniegelenksluxation. Trauma Berufskrankh 2017; 19: 289-296. doi:10.1007/s10039-017-0314-2

[3] Ridley T], Cook S, Bollier M et al. Effect of body mass index on patients with multiligamentous knee injuries. Arthroscopy 2014; 30: 1447-1452. doi:10.1016/j.arthro.2014.05.035

[4] Seroyer ST, Musahl V, Harner CD. Management of the acute knee dislocation: the Pittsburgh experience. Injury 2008; 39: 710-718. doi:10.1016/j.injury.2007.11.022

[5] Green NE, Allen BL. Vascular injuries associated with dislocation of the knee. J Bone Joint Surg Am 1977; 59: 236-239

[6] Mills WJ, Barei DP, McNair P. The value of the ankle-brachial index for diagnosing arterial injury after knee dislocation: a prospective study. J Trauma 2004; 56: 1261-1265
[7] Barnes C], Pietrobon R, Higgins LD. Does the pulse examination in patients with traumatic knee dislocation predict a surgical arterial injury? A meta-analysis. J Trauma 2002; 53: 1109-1114. doi:10.1097/01.TA.0000025792.36332.D7

[8] Levy BA, Dajani KA, Whelan DB et al. Decision making in the multiligament-injured knee: an evidence-based systematic review. Arthroscopy 2009; 25: 430-438. doi:10.1016/j.arthro. 2009.01.008

[9] Frosch KH, Preiss A, Heider S et al. Primary ligament sutures as a treatment option of knee dislocations: a meta-analysis. Knee Surg Sports Traumatol Arthrosc 2013; 21: 1502-1509. doi:10.1007/s00167-012-2154-8

[10] Heitmann M, Gerau M, Hötzel ] et al. [Ligament bracing-augmented primary suture repair in multiligamentous knee injuries]. Oper Orthop Traumatol 2014; 26: 19-29. doi:10.1007| s00064-013-0263-2

[11] Richter M, Lobenhoffer P, Tscherne H. [Knee dislocation. Longterm results after operative treatment]. Chirurg 1999; 70: 1294-1301

[12] Preiss A, Giannakos A, Frosch K-H. Minimal-invasive Augmentation des medialen Kollateralbandes mit autologen Hamstringsehnen bei chronischen Instabilitäten am Kniegelenk. Oper Orthop Traumatol 2012; 24: 335-347. doi:10.1007| s00064-012-0164-9

[13] Domnick C, Frosch KH, Raschke M] et al. Kinematics of different components of the posterolateral corner of the knee in the lateral collateral ligament-intact state: a human cadaveric study. Arthroscopy 2017; 33: 1821-1830.e1. doi:10.1016/j. arthro.2017.03.035

[14] Stannard JP, Brown SL, Farris RC et al. The posterolateral corner of the knee: repair versus reconstruction. Am J Sports Med 2005; 33: 881-888. doi:10.1177/0363546504271208

[15] LaPrade RF, Johansen S, Agel J et al. Outcomes of an anatomic posterolateral knee reconstruction. J Bone Joint Surg Am 2010; 92: 16-22. doi:10.2106/JBJS.I.00474

[16] Arciero RA. Anatomic posterolateral corner knee reconstruction. Arthroscopy 2005; 21: 1147. doi:10.1016/j.arthro.2005. 06.008

[17] Bobrich E, Haupt C, Grass R et al. Tibiakopffraktur und Luxationsfraktur. Trauma Berufskrankh 2009; 11: 154-159. doi:10.1007/s10039-009-1504-3

[18] Krause M, Müller G, Frosch K-H. Chirurgische Zugänge bei Tibiakopffrakturen. Unfallchirurg 2018. doi:10.1007/s00113018-0515-6

[19] Frosch KH, Akoto R, Drenck T et al. Arthroscopic popliteus bypass graft for posterolateral instabilities of the knee: a new surgical technique. Oper Orthop Traumatol 2016; 28: 193203. doi:10.1007/s00064-015-0432-6

\section{Bibliografie}

DOI https://doi.org/10.1055/a-0718-4320

Online-publiziert 15.01.2019| OP-JOURNAL 2019; 34: 163170 ๑ Georg Thieme Verlag KG Stuttgart · New York ISSN 0178-1715 